\title{
An investigation of Chromatium vinosum high-potential iron-sulfur protein by EPR and Mossbauer spectroscopy; evidence for a freezing-induced dimerization in $\mathrm{NaCl}$ solutions
}

\author{
W. Richard Dunham ', Wilfred R. Hagen ', James A. Fee ',*, Richard H. Sands ', \\ James B. Dunbar ${ }^{2}$ and Christine Humblet ${ }^{2}$ \\ 'Biophysics Research Dicision, Unicersity of Michigan, Ann Arbor, MI (U.S.A.) and '2 Parke-Dat is Pharmaceutical Research Ditision \\ Warner-Lambert Co., Ann Arhor. MI (U.S.A.)
}

(Received 27 March 199:)

Key words: High potential iron-sulfur protein: EPR: Spectroscopy: Mossbauer spectroscopy: Cuhane: Structure: g-Strain: Salt effect

The high-potential iron-sulfur protein (HiPIP) from Chromatium cinosum contains a cubane prosthetic group that shuttles between the $[4 F e-4 S]^{3+, 2+}$ states. We find that the EPR spectra from this protein can be explained as a sum of two components, a major one with $g=2.02 ; 2.04 ; 2.12$, and a minor one with $g=2.04 ; 2.07 ; \sim 2.13$. In the presence of 0.1-2.0 M NaCl, freezing induces polymerization of the protein (presumably dimers), which is detected as intercluster spin-spin interaction in the EPR. The observed spin-spin interactions are interpreted as being due to two very similar dimeric structures in an approx. $1: 2$ ratio. Computer simulation of the $X$ - and Q-band EPR spectra shows that the z-components of the g-tensors in each dimer pair must be co-linear, with center-to-center distances between the clusters of $\sim 13 \AA$ and $\sim 16 \AA$. Inspection of possible dimeric structures of $C$. cinosum HiPIP by standard molecular graphics procedures revealed that the $\mathrm{Fe} / \mathrm{S}$ cluster is exposed toward a flattened surface and is accessible to solvent. Moreover, the Fe / S clusters in two HiPIP molecules can easily achieve a center-to-center distance of $\sim 14 \AA$ when approaching along a common 3-fold axis that exiends through the $S 4$ sulfur atom of the cubane; the z-component of the EPR g-tensor is co-linear with this symmetry axis.

\section{Introduction}

The [4Fe-4S] prosthetic group is a common arrangement of iron and acid-labile sulfur ions in biological systems and its cubane structure has been characterized by a wide variety of chemical and spectroscopic techniques [1-3]. The purple phototrophic bacteria are an important source of $[4 \mathrm{Fe}-4 \mathrm{~S}]$ cluster-containing proteins, particularly the so-called high potential iron proteins (HiPIPs), which have reduction potentials for the $[4 \mathrm{Fe}-4 \mathrm{~S}]^{3+2+}$ redox couple in the range 50 to $450 \mathrm{mV}$ [4]. Fukumori and Yamanaka [5] have suggested a

\footnotetext{
* Present address: Biochemistry Section and Stable Isotope Resource. Isotope and Structural Chemistry Group. Los Alamos National Laboratory, Los alamos, NM 87545. U.S.A.

Abbreviations: HiPIP, high-potential iron protein.
}

Correspondence: W. Richaid Dunham. Biophysics Research Division, Unversity of Michigan. 2200 Bonisteel Bhd. Ann Arbor, MI 48109. U.S.A. physiological role for these proteins in the oxidation of thiosulfate. Whatever their function, these proteins have played an important role in the development of the field of $\mathrm{Fe} / \mathrm{S}$ biochemistry. The HiPIP from Chromatium tinosum, which is a small, readily available prote'n containing a single [4Fe-4S] cluster, has received the most attention. After its initial recognition and purification by Bartsch [6], its amino acid sequence was determined [7], and its three-dimensional structure was deduced by $X$-ray crystallography [8.9]. This revealed the cubane arrangement of the $4 \mathrm{Fe}$ and $4 \mathrm{~S}$ atoms and showed that the cluster was coordinated to 4 cysteine thiolate groups. The amino acid sequences of additional HiPIPs have been determined [10], but there is only one three-fimensional structure in the literature.

Considerable work has been done on the spectral and magnetic properties of the $C$. linosum HiPIP. The reduced form of the protein. [4Fe-4S] $]^{2}$. has an $S=0$ ground state [11]. but $H$-NMR studies have revealed $S_{\text {eff }}>0$ at ambient temperatures indicating the pres- 
ence of populated states having $S>0[12]$. The cxidized protein, $[4 \mathrm{Fe}-4 \mathrm{~S}]^{3+}$, has an $S=1 / 2$ ground state, as deduced from magnetic susceptibility studies [:2]; however, EPR studies of the oxidized protein revealed an unexpected heterogeneity [13]. In 1975 Antanaitis and Moss[14] showed that the additional features in the EPR signal were independent of microwave frequency and thus arise from 'real $g$-values.' These authors suggested that the EPR spectrum of oxidized $C$. vinosum HiPIP is a "superposition of three distinct signals, namely, two principal signals of eoual weight, one reflecting axial and the other rhorioic symnistrv, and a third nearly isotropic minority somponent". It was further argued that the two majo: EPR components arise from distinct pairs of $\mathrm{Fe}$ atons, which the effective spin "samples at a rate $\sim 10^{7}-10^{8} \mathrm{~Hz}$ ". This spin distribution scheme was consistent with then extant NMR [12] and Mossbauer data [15,16], and there the proposal has largely rested in the intervening years. Here we take up and expand on the theme of active site heterogeneity using EPR and Mossbauer spectroscopy to examine samples of $C$. tinosum HiPIP subjected to different solvents, solutes, degree of oxidation and mode of freezing. These studies result in an interpretation different from that of the earlier investigators [14].

\section{Materials and Methods}

\section{Materials}

1. Iromatium tinosum cells were obtained from the American Type Culture Collection (ATCC \#17899) and grown photosynthetically, using sulfide and thiosulfate as electron donors [17]. High potential iron protein was purified and spectrally characterized by the procedures of Bartsch [18]. The purity ratio $\left(A_{283} / A_{388}\right)$ of our samples was typically 2.50 and they showed the presence of only minor contaminants ( $2 \%$ ) that stained by Coomassie blue upon gel electrophoresis. All chemicals used in this work were of the highest quality commercially available and were used without further manipulation. For some experiments, C. tinosum was grown in the presence of ${ }^{15} \mathrm{NH}_{4} \mathrm{Cl}$ to replace all ${ }^{14} \mathrm{~N}$ atoms of the cell with ${ }^{15} \mathrm{~N}$; and with enriched ${ }^{57} \mathrm{Fe}$ to obtain HiPIP suitable for Mossbauer studies.

\section{Methods}

EPR spectroscopy at $9 \mathrm{GHz}$ was carried out on a Varian E-112 spectrometer. Microwave frequency was determined using a Hewlett-Packard 5340A frequency counter. Field calibration was accomplished with a Systron-Donner Model 3193 digital NMR gaussmeter. EPR spectroscopy at $35 \mathrm{GHz}$ was carried out on a Varian V-4500 spectrometer equipped with a V-4561
$35 \mathrm{GHz}$ microwave bridge. Field modulation was at 100 $\mathrm{kHz}$ on both spectrometers. Sample temperature was maintained using a homemade, gas phase liquid helium transfer line and quartz dewar inset. Sample temperature measurements were made using a $0.1 \mathrm{~W}$ Allen Bradley carbon composition resistor calibrated at liquid helium, liquid nitrogen and ice-water and placed directly below the sample tube.

To determine the fraction of oxidized protein, HiPIP samples were mixed with known amounts of $K_{3} \mathrm{Fe}$ $(\mathrm{CN})_{6}$, frozen in liquid nitrogen, and the EPR spectra recorded at $16+1 \mathrm{~K}$ under non-saturating microwave powers; $\mathrm{Cu}^{2+}$ EDTA was used as a secondary spin quantitation standard. Spin concentrations were determined by the cut-and-weigh method [19]. The data yielded a value of $[S=1 / 2] /[4 \mathrm{Fe}-4 \mathrm{~S}]=1.00 \pm 0.05$.

Rapid freeze-quench experiments were done using the method of Bray[20] as modified by Ballou [21] on an apparatus designed and built by Professor David Ballou of The University of Michigan.

Mossbauer spectra were obtained with a home-built spectrometer and associated hardware as described elsewhere $[22,23]$. Spectra presented have been corrected for source lineshape and converted from transmission to absorption spectra. Previous examples of this approach to Mossbauer spectroscopy of biological macromolecules, including theoretical simulation of experimental spectra, are given in Refs. 24 and 25 .

Simulation of experimental, frozen solution, $S=1 / 2$ EPR spectra were based on the previously developed statistical theory of g-strain[26]. The g-strain simulator was operated in f:ll positive correlation mode, and the simulations reported here are comparable to those reported previously. See Ref. 27 for a review of g-strain in biological systems. Simulation of spectra resulting from spin-spin interactions was achieved using the method described by Schepler et al.[28] witi an extension of the computer code to permit for g-strain in full positive correlation mode (Dunham et al., unpublished data).

Molecular modeling studies were undertaken with the polymer module available in the SYBYL [29] software package. The rrvstal structure [8,9] of the Chromatium HiPIP (entry 'HIP) was retrieved from the Brookhaven National Laboratory Protein Data Bank. A geometric plane including the iron atoms $\mathrm{Fe} 2$ to $\mathrm{Fe} 4$ was used to construct a $14 \AA$ long normal through the sulfur atom $\mathrm{S}^{*} 4$, thus defining the cubane 3 -fold rotation axis of interest in this study. This molecular structure was then duplicated to assemble the initial dimeric model. Molecular modeling techniques utilizing the interactive graph: manipulation capabilities available with SYBYL [29] were used to align the two monomers along tii ild axis extending through $S^{*} 4$. Both Van ver Waals and overall hydropathy/electrostatic com$v^{\prime} \quad$ arity checks were applied to select the respec- 
tive monomer orientation within the dimeric association.

\section{Results and Discussion}

The EPR spectrum of oxidized $C$. vinosum HiPIP, recorded in a pH 8 solution of $20 \mathrm{mM}$ Tris- $\mathrm{HCl}$ is shown in Fig. 1A. It is characterized by a prominent low-field ramp; a broad, asymmetric $g_{z}$ feature; a distinct 'bump' near $g=2.08$; and a broad but clearly asymmetric $g_{x, y}$ region. This was the spectrum analyzed by Antanaitis and Moss [14].

In the course of purifying samples of $C$. cinosum HiPIP for detailed physical studies, it was noted that the EPR spectrum was distinctly modified by $\mathrm{NaCl}$ concentrations in the $\sim 0.2-2 \mathrm{M}$ range. This characteristic 'high salt' spectrum is shown in Fig. 1E. Spectra A through $\mathrm{E}$ show the effect of increasing $\mathrm{NaCl}$ from 0 to $0.25 \mathrm{M}$ in a Tris-Cl buffer at $\mathrm{pH} 8$. Under this perturbation, the low-field ramp is eliminated, novel structure appears in the $g_{2}$ region, and rhombicity is resolved in the $g_{x, y}$ region. This transformation was observed with many independent preparations of proteins and was reported in preliminary form in 1976 at

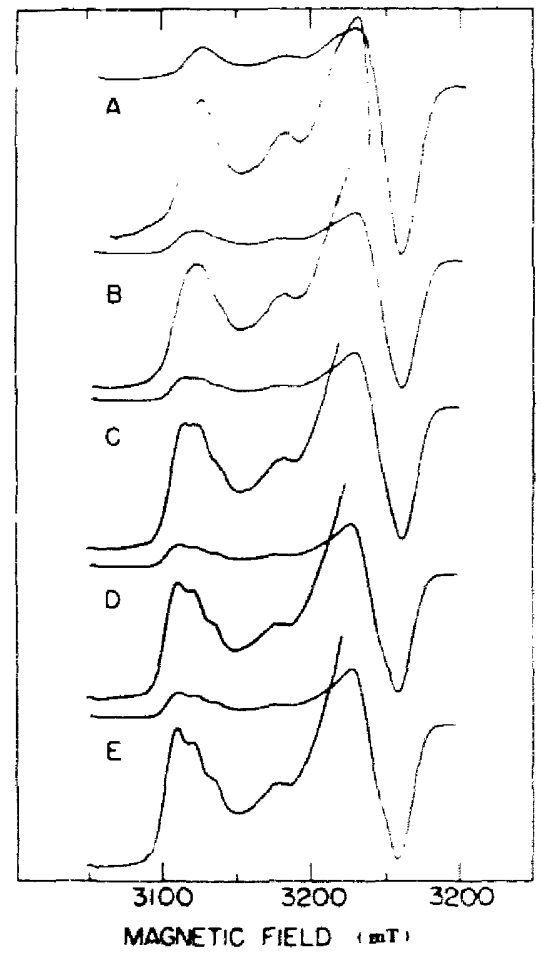

Fig. 1. Titration of $330 \mu \mathrm{M} C$. linosum HiPIP in $20 \mathrm{mM}$ Tris HCl, $\mathrm{pH}$ 8.0. with increasing concentrations of $\mathrm{NaCl}$. namely. (trace A). 0 $\mathrm{mM}$ : (B) $25 \mathrm{mM}$ : (C) $50 \mathrm{mM}$ : (D) $75 \mathrm{mM}$; (E) $100 \mathrm{mM}$. The insets are scaled to give approximately the same vertical range on the figure. The EPR running conditions are: temperature. 16K: microwave power. $300 \mu \mathrm{W}$; modulation amplitude. $0.5 \mathrm{mT}$ : sweep time. $5 \mathrm{~min}$.

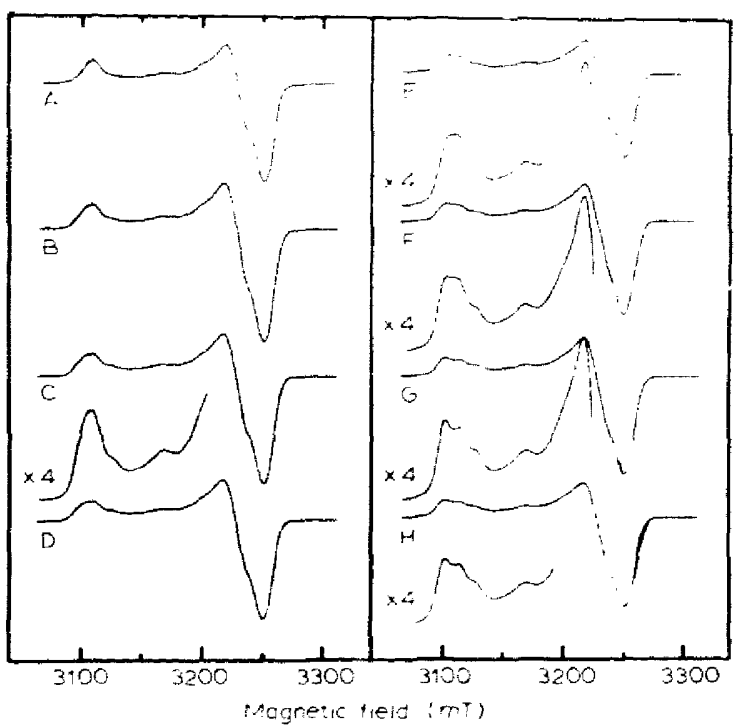

Fig. 2. EPR oxidative titration of $C$. linosum HiPIP with potassium ferricyanide in the presence of $0.2 \mathrm{M} \mathrm{NaCl}$. The protein was 1.02 $\mathrm{mM}$ in $20 \mathrm{mM}$ Tris-HCl. pH 8.0. The amount of added equivalents of ferricyanide and the percentage of oxidized HiPIP, as determined by double integration, are: (trace A) $0.24,29 \%$; (B) $0.49,56 \%$ : (C) 0.62 , 66\%: (D) $0.70,82 \%$; (E) $0.7 \%, 88 \%$; (F) $0.86,97 \%$; (G) $1.00 .100 \%$; (H) $1.81,100 \%$. The EPR conditions were as for Fig. 1 .

the VIIth International Conference on Magnetic Resonance in Biological Systems [30].

The effect of $\mathrm{NaCl}$ seems to be specific. We examined the following matrix of cations $\left(\mathrm{Li}^{+}, \mathrm{Na}^{+}, \mathrm{K}^{+}\right.$and $\left.\mathrm{NH}_{4}^{+}\right)$and anions $\left(\mathrm{Cl}^{-}, \mathrm{NO}_{3}^{-}, \mathrm{ClO}_{4}^{-}\right.$and $\left.\mathrm{SO}_{4}^{2-}\right)$, excepting $\mathrm{KClO}_{4}$, and found that salts in general had only minor effects on the typical 'low salt' EPR spectrum; only the $\mathrm{Na}^{+}$plus $\mathrm{Cl}^{-}$combination of ions had an interesting influence on the EPR spectrum. The potential effect of $\mathrm{pH}$ on the "high salt" spectrum was not examined. However, Antanaitis and Moss [14] demonstrated that the apparent heterogeneity in the EPR spectrum of the 'low salt' spectrum was independent of $\mathrm{pH}$ in the 2 to 11 range.

The effect of $\mathrm{NaCl}$ is further characterized by an oxidative titration. Fis. 2 shows the X-band EPR spectrum of HiPIP in $0.2 \mathrm{M} \mathrm{NaCl}$ at increasing fractions of oxidation $(25$ to $100 \%)$. It is noteworthy that the spectrum recorded at $25 \%$ oxidation lacks the low-field ramp and the structure in the $g_{2}$ region, but does show the same resolution as the fully oxidized sample in the $g_{x, y}$ region. As the fraction of oxidized molecules in creases, one observes the apparent incremental appearance of the unusual structure around $g_{z}$. A similar experiment at low salt conditions revealed no qualitative differences in the X-band EPR spectrum at different levels of oxidation; all spectra were similar to the spectrum of Fig. 1A (data not shown).

To test the possibility of magnetic hyperfine interactions as the cause of the structure in the "high salt" 
EPR spectrum, we carried out a number of isotope substitutions. Solvent was exchanged from ${ }^{1} \mathrm{H}_{2} \mathrm{O}$ to ${ }^{2} \mathrm{H}_{2} \mathrm{O} ; \mathrm{Na}^{35} \mathrm{Cl}$ and $\mathrm{Na}^{37} \mathrm{Cl}$ were compared; and a complete exchange of ${ }^{15} \mathrm{~N}$ for ${ }^{14} \mathrm{~N}$ was effected by growing the bacteria on an ${ }^{15} \mathrm{~N}$-enriched nitrogen source. These substitutions caused no differences in the EPR spectrum. Finally, we recorded the spectrum at $35 \mathrm{GHz}$ and obtained no evidence for nuclear hyperfine interaction as the origin of the structure around $g_{7}$ in the 'high salt' EPR spectrum (see below).

The 'high salt' EPR spectrum was converted to the typical 'low salt' spectrum by addition of excess of salts, diluents or miscible organic solvents. By increasing the concentration of $\mathrm{NaCl}$ above $2 \mathrm{M}$, the characteristic structure in the EPR was lost. Similarly, the presence of a high concentration of serum albumin ( 60 $\mathrm{mg} / \mathrm{ml}$ ) precluded deveiupinent of the unique signa!. Including solvents such as ethylene glycol, glycerol or ethanol (at $\sim 50 \% \mathrm{v} / \mathrm{v}$ ) led to appearance of the 'low salt' spectrum (data not shown). Ethanol at $50 \%$, however, induced a better resolved'low salt' spectrum, which is shown in Fig. 5D and discussed further below.

Knowing that diluents which change the global properties of the solvent, such as ethylene glycol, obviated the 'high salt' effect led us to suspect some type of protein-protein association. This idea was tested in a rapid freeze-quench study. In this experiment, a concentrated solution of fully oxidized HiPIP in $5 \mathrm{mM}$ Tris- $\mathrm{HCl}$ was mixed with an equal volume of similarly buffered $1 \mathrm{M} \mathrm{NaCl}$, frozen in cold isopentane in less than $10 \mathrm{~ms}$ and packed into an EPR tube. The X-band EPR spectrum is shown in Fig. $3 A$. The sample was subsequently thawed, refrozen and the spectrum of Fig. 3B recorded. Spectra obtained at freezing times of $\sim 15$ and $\sim 50 \mathrm{~ms}$ were also examined. Visual inspection of the resulting EPR spectra suggested a time-dependent appearance of the 'high salt' component, but this was incomplete even at $50 \mathrm{~ms}$ (data not shown). At the fastest time of freezing, however, the spectrum appears to be primarily that of the 'low salt' form (Fig. 3A). These observations suggest that solutions of oxidized $C$. ıinosum HiPIP are modified by freezing and this process has a characteristic time of tens of milliseconds. The idea that some type of protein-protein interaction is occurring will be discussed below.

We make the following conclusions regarding heterogeneity in the EPR spectrum of oxidized $C$. $v i$ nosum HiPIP: first there is an inherent heterogeneity, typified by the spectra obtained in 'low salt', $50 \%$ ethanol' and 'high salt, rapidly frozen' conditions. Second, the inherent heterogeneity is specifically altered by slow freezing in the presence of 'high salt'. This change is characterized by the EPR spectrum of the $25 \%$ oxidized sample shown in Fig. 2A. Third, 'high salt' induces additional heterogeneity in fully oxidized samples, typified by the 'slowly frozen, high salt' spec-

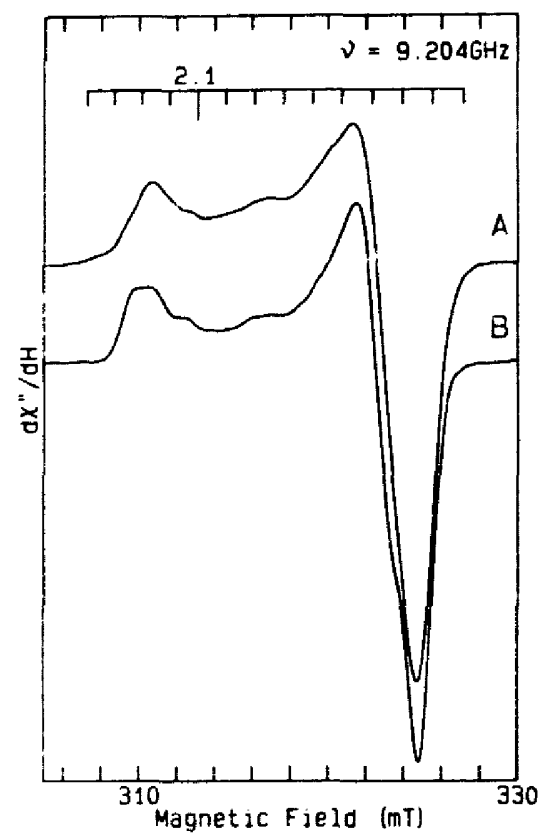

Fig. 3. Effects of the mode of freezing on the EPR line shape of $C$. inosum HiPIP in the presence of $\mathrm{NaCl}$. The protein. $117 \mu \mathrm{M}$ in 5 $\mathrm{mM}$ Tris-HCl, pH 8.0. was rapidly $1: 1$ mixed with $1 \mathrm{M} \mathrm{NaCl}$ in $5 \mathrm{mM}$ Tris-HCl. Aft $3+10 \mathrm{~ms}$ aging the mixture was squirted into isopentane $\left(-140^{\circ} \mathrm{C}\right)$ and collected into an EPR tube. The resulting spectrum is given in trace $A$. After thawing and re-freezing slowly in liquid nitrogen, the spectrum of trace $B$ was obtained. EPR conditions: microwave frequency, $9.20 \mathrm{GHz}$; microwave power, $2 \mathrm{~mW}$; modulation amplitude, $0.8 \mathrm{mT}$; temperature, $19 \mathrm{~K}$.

trum of Fig. 1E. There are therefore at least three distinct types of EPR spectra, and these are analyzed in subsequent paragraphs.

\section{Mossbauer studies}

It is important to ascertain the extent to which the $\mathrm{NaCl}^{-}$induced changes reflected in the EPR spectra arise from differences at the individual iron atoms. High resolution ${ }^{57} \mathrm{Fe}$ Mossbauer spectroscopy is well suited to address this concern. In Fig. 4A we compare the Mossbauer spectra of fully oxidized, slowly frozen C. tinosum HiPIP in the 'low salt' and in the 'high salt' $(0.2 \mathrm{M} \mathrm{NaCl})$ condition. The spectra are similar to those previously published by others $[15,16,31]$ and are indistinguishable between the two conditions. A similar experiment is presented in Fig. 4B, in which two HiPIP samples, now both in the presence of $0.2 \mathrm{M} \mathrm{NaCl}$, are compared, one being only $25 \%$ oxidized while the other one is fully oxidized. The spectrum of the fully oxidized sample is reduced a factor of three with respect to the absorption axis for ease of comparing the two line shapes in detail. With the restrictions in mind that the spectrum of partially oxidized HiPIP has a $3 \times$ higher noise level and is overshadowed in the central part by the spectrum from $S=0$, reduced protein, these two preparations also give identical Mossbauer spectra. Therefore, the 'high salt' condition is 


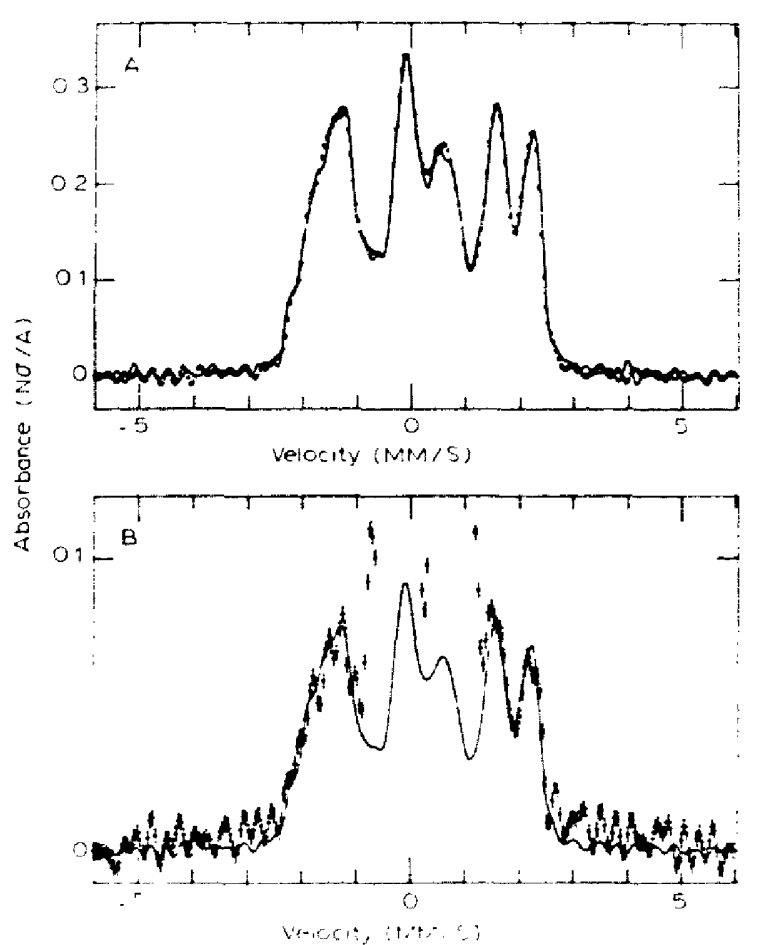

Fig. 4. $\mathrm{NaCl}$ has no effect on the Mossbauer spectral properties of $C$ linosum HiPlP. The Mossbauer spectra taken at $4.2 \mathrm{~K}$ and $0.1 \mathrm{~T}$ applied magnetic field for samples of various salı and cxidation state are superimposed for comparison. In Part $A$ the 'low salt' form 'solid line) is superimposed on the 'high salt' form (crosses). No rescaling of the data has been performed. In Part B. the spectrum of the "high salt. $25 \%$ oxidized' form is superimposed by a spectrum of the "high salt. $100 \%$ oxidized' sample (solid line). Points are omitted from the 'partially oxidized' spectrum when the quadrupole doublet of the reduced $C$. tinosum HiPIP molecules went off-scale. The size of the $100 \%$ oxidized' spectrum has been reduced a factor of three to facilitate comparison.

not induced by changes at an individual iron atom. The question of minority contributions to the Mossbauer spectrum is discussed below.

\section{Analysis of the EPR spectra}

Inherent heterogeneity. The spectra in Fig. 5 summarize the data and curve fitting efforts for oxidized $C$. vinusum HiPIP recorded under three different solutions and characterize what we call inherent heterogeneity. Spectrum $5 \mathrm{~A}$ is the stick representation of the simulation by Antanaitis and Moss [14] to a spectrum identical to Spectrum 5B. When these sticks are convolved with a Gaussian lineshape and added in a 1:1:0.2 proportion, they lead to a reasonable representation of the experimental spectrum (see Fig. 2 of Ref. 14). The solid lines ir: spectra B-D are experimental spectra of: B, 'low salt', fully oxidized; C, 'high salt'. $25 \%$ oxidized; and D, $50 \%$ ethanol $100 \%$ oxidized HiPIP. We have simulated each of these spectra using

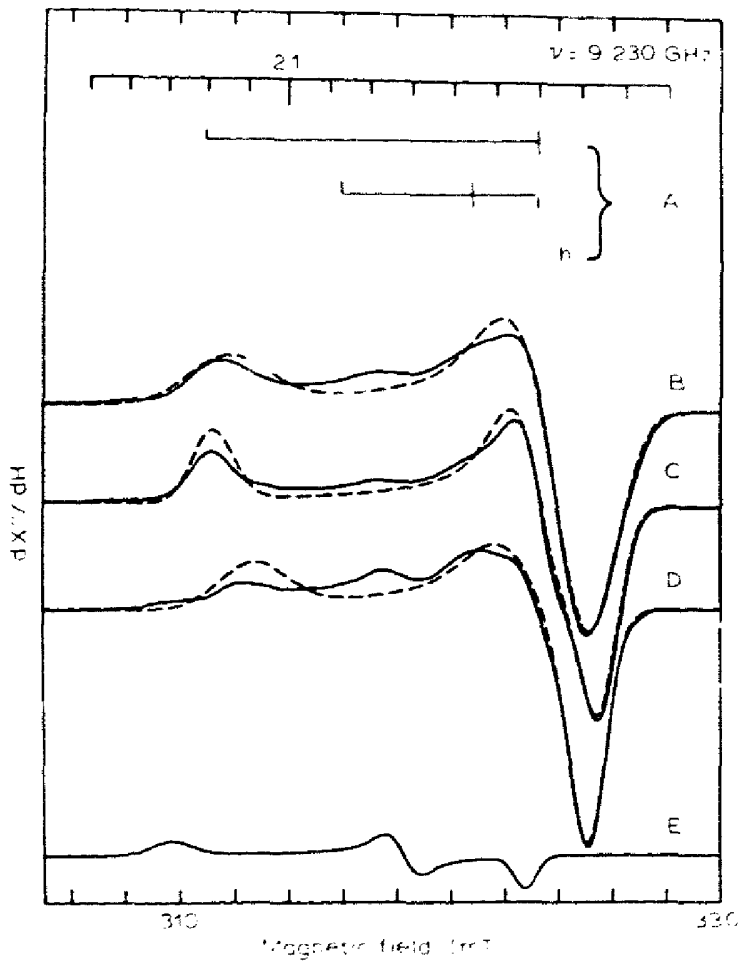

Fig. 5. Comparison of $C$. linosum HiPIP spectra under varying conditions. The set of three bars in Part $A$ represents the previous interpretation by Antanaitis and Moss [18]. Part B shows the low salt, oxidized' form (Fig. 1A) superimposed by its one-component computer fit (dashed line). Part $\mathrm{C}$ is the 'high salt. $25 \%$ oxidized' form (Fig. 2A) superimposed by its one-component computer fit (dashed line). Part D is the ' $50 \%$ EtOH. oxidized' form superimposed by its one-component computer fit (dashed line). Part $E$ is a computer simulation of the minority component visible in the experi. mental trace of Part D. The spectral fitting parameters are shown in Table 1.

a one-component model with the parameters given in Table I. The results are shown in Fig. IB-D as spectral overlays (dotted lines).

\section{TABLE I}

EPR parameters of tho $S=1 / 2$ ground states from C: Vinostum HiPIP in different frozen matrices

The dimers are comprised of two identicat. colinear $S=1 / 2$ spin systems. $r$ (tho: intercluster vector) is along the - -axes of both g-tensors. The $\sigma_{t}$ are linewidth cone-half the distance between the derivative extremal in $g$-value units.

\begin{tabular}{|c|c|c|c|c|c|c|}
\hline & $\begin{array}{l}\text { Low } \mathrm{NaCl} \\
\text { monomer }\end{array}$ & $\begin{array}{l}\text { High } \mathrm{NaCl} \\
\text { monomer }\end{array}$ & $50 \mathrm{EtOH}$ & Minority & $\begin{array}{l}0.2 \mathrm{M} \mathrm{N} \\
\text { dimer }\end{array}$ & $\mathrm{NaCl}$ \\
\hline$g_{k}$ & 2.025 & 2.023 & 2.027 & 2.041 & 2.024 & 2.024 \\
\hline$g$ & 2.037 & 2.038 & 2.039 & 2.071 & 2.038 & 2.038 \\
\hline$g$ & 2.115 & 2.118 & 2.108 & 2.129 & 2.114 & 2.118 \\
\hline$\sigma_{k}$ & 0.007 & 0.005 & 0.005 & 0,003 & 0.004 & 0.004 \\
\hline$\sigma_{1}$ & 0.006 & 0.006 & 0.010 & 0.004 & 0.007 & 0.007 \\
\hline$\sigma$ & 0.004 & 0.006 & 0.010 & 0.006 & 0.005 & 0.005 \\
\hline$\because$ & 98 & 97.4 & 9.3 .7 & - & 33 & 67 \\
\hline$r(A)$ & - & - & - & - & 13 & 16 \\
\hline
\end{tabular}


There are two major reasons for the lack of fit in these simulations. The first is seen by comparing the data in Fig. 5B-D. There is obviously a minor component in these spectra, which varies in amount depending on the solvent and salt parameters. In Fig. 5D, from the 'glassy' sample. the minor component is large enough that all three $g$-values are visible, and its simulated shape is shown is Fig. $5 \mathrm{E}$ using the parameters in Table 1. Although the cntire spectrum of the minor component is not clear in Fig. 5B and C, the midsweep turning points are clear in all three spectra. By trial and error fitting of this part of the minority spectrum, we were able to quantitate the minority component as $2.0,2.3$ and $7.4 \%$, respectively, of Fig. 5B, C and D. These numbers are obviously in disagreement with the $50 \%$ values in the previous analysis [14]. A second spectral fitting problcm concerns the type of ' $\mathrm{g}$-strain' in these spectra. We have found that EPR spectra from several other HiPIP's can be fit 'through the dots' by our simulation programs (our unpublished observations). That $C$. linosum HiPIP cannot be fit in this way seems to derive from some special properties of this protein's freezing dynamics as will be considered in the following discussion.

The question of whether the minority component seen in the EPR spectra can be detected by Mossbauer spectroscopy is addressed by the data of Fig. 6. At high temperature, where the magnetic hyperfine splittings are averaged to zero, each iron atom of the cubane will contribute only two lines to the Mossbauer spectrum as determined by its quadrupole splitting, its isomer shift, and its linewidth. As a minimal hypothesis we have assumed that the eight contributing lines have equal intensity and width. Simulated (dots) and experimental (crosses) spectra of 'high salt' (A) and 'low salt' (B) samples are shown in Fig. 6. There are two important points: first, there is essentially no difference between the two experimental spectra; second, very small deviations between experimental and simulations seen near $0.9,0.4,0.2$ and $-0.2 \mathrm{~mm} / \mathrm{s}$ arise from only $0.5 \%$ of the total iron in the sample. If this residual intensity is assigned to one iron atom in $2 \%$ of the HiPIP molecules, then a good correspondence exists with the quantitation of the minority EPR signal (Fig. 5 and Table I). However, because of the paucity of signal, we are unable to uniquely describe its Mossbauer spectrum.

Induced heterogeneity. X- and Q-band EPR spectra of fully oxidized, 'high salt/slow frozen' $C$. i inosum HiPIP are shown in Fig. 7 ; the spectra are shown on a similar g-scale to facilitate comparison. The multiple lines in the low-field region of the $X$-band spectrum (Fig. 7A) are clearly not the result of multiple g-tensors since this regiori of the Q-band spectrum (Fig. 7B) does not show this structure. The multiple peaks in the low-fic'd region of Fig. 7A are thus likely to result from

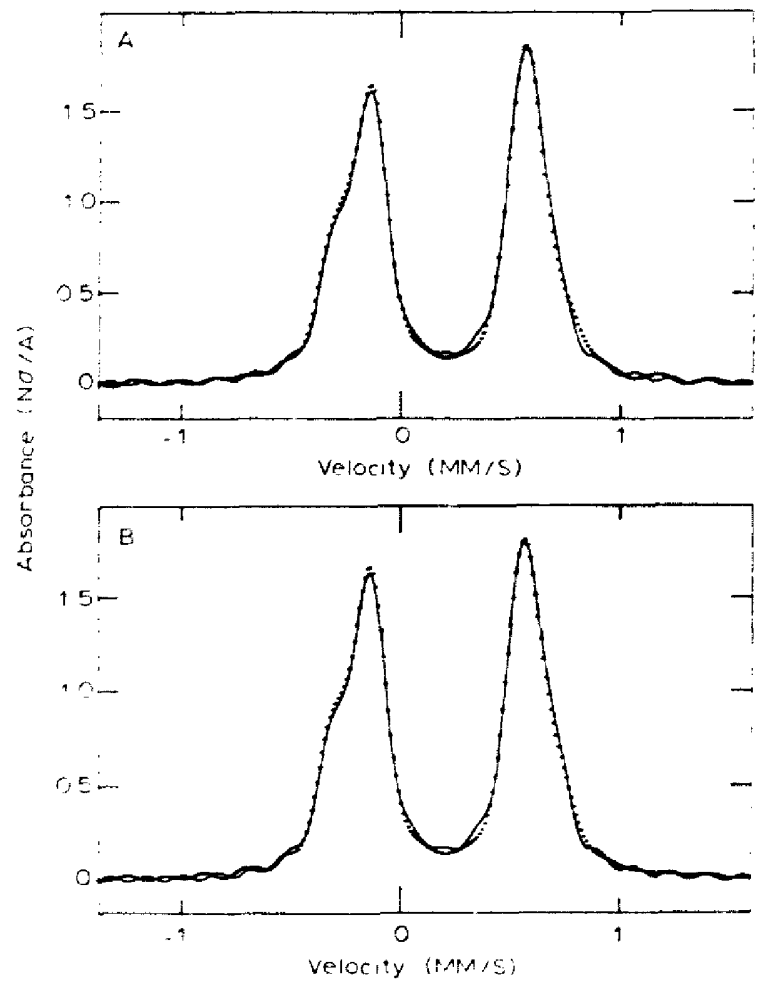

Fig. 6. A single cuhane can account for at least $98 \%$ of the iron in $C$. rinosum HiPIP regardless of salt concentration. The Mossbauer spectra (160)K. zero applied magnetic field) of $C$. tinosum HiPIP at high salt (Part $A$ ) and low salt (Part B) are shown superimposed by computer fits based on eight lines of equal magnitude and linewidth $(0.15 \mathrm{~mm} / \mathrm{s})$.

some kind of hyperfine interaction. The results of the isotopic exchange experiments and the Mossbauer data argue against this splitting being due to a nuclear hyperfine interaction. By elimination, therefore, we are left with an exchange interaction (direct or dipolar) as the most likely explanation for the fine structure. In the following paragraphs, we develop the hypothesis that, upon slow freezing, $\mathrm{NaCl}$ induces formation of a unique interaction between HiPIP molecules that leads to the fine structure.

The spectra in Fig. 7 are overlaid by two simulations (dashed lines) whose parameters are available in Table I under the columns for the 'high salt' dimers. Both simulations (at $\mathrm{X}$ - and $\mathrm{Q}$-band) have the same spin Hamiltonian parameters. These parameter values imply that the $S=1 / 2$ spin systems of two identical HiPIP molecules are in magnetic dipole-dipole interaction with the intercluster direction along the $z$-axis of each cluster. There are two configurations for this interaction (with slightly different $g_{\mathrm{z}}$ values) corresponding to two intercluster distances of 13 and $16 \AA$ : the dimer with $16 \AA$ distance being twice as probable as the $13 \AA$ situation. In brief, our justification of this picture is that we have tried all of the other possible models that are consistent with the physics of the 

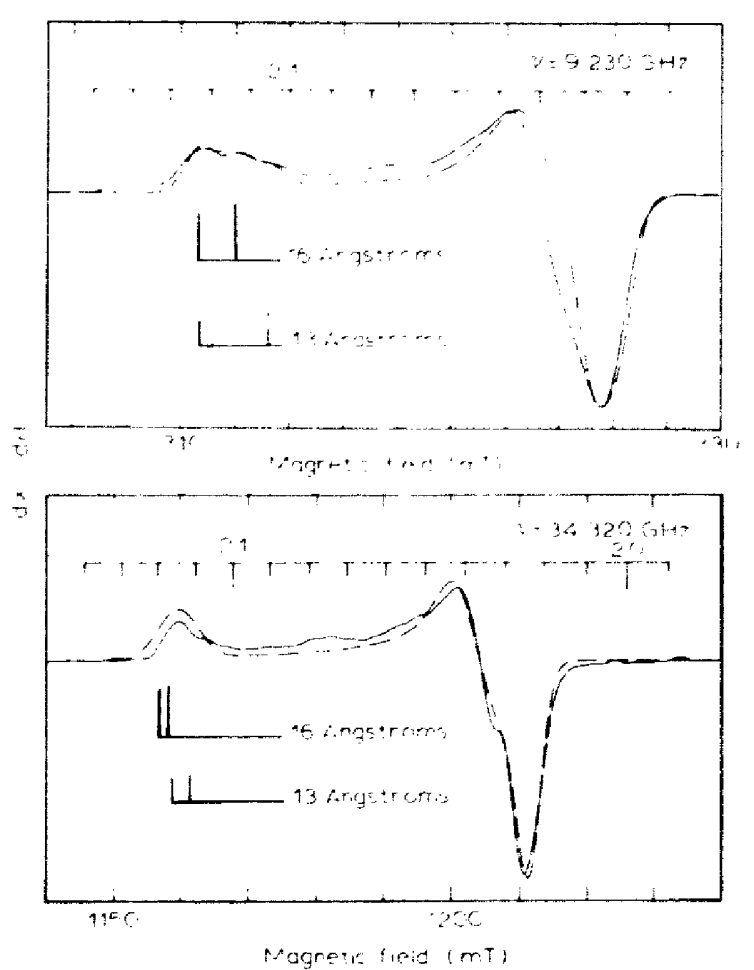

Fig. 7. X-and Q-band spectra of $100 \%$ oxidized. C. rinosum HiPIP in $0.2 \mathrm{M} \mathrm{NaCl}$. Both spectra are fit (dashed lines) by the same dipole-dipole, interacting one-component spin-spin model (see Table I for paranters). The stick spectral inserts denote the positions and relative intensities of the component spectra in the $g$, region. Sample preparation and X-band running conditions as in Fig. 1. The Q-band spectrum was recorded at $18 \mathrm{~K}$. field sweep: 200 $\mathrm{mT} / \mathrm{min}$, field modulation amplitude: $0.2 \mathrm{mT}$, microwave power: $0.7 \mu \mathrm{W}$.

problem, and none of these models is allowed by the data. Our argument requires an understanding of the range of possibilities of EPR spectra from two weakly interacting paramagnets as constrained by the experimental details.

Each spin-spin interaction (dimer) is assumed to involve two isolated $S=1 / 2$ spins (each denoted by g-tensor). A set of interaction parameters describes the direct exchange (a $J$-value) and the dipole-dipole interaction (a distance and direction for the intersystem vector and a set of Euler angles to give the orientation of one g-tensor relative to the other). Linewidth is treated by extending our g-strain theory to the interaction situation. Therefore, the mathematics for spectral simulation of these EPR spectra includes a large number of possible parameters. We can limit these possibilities by comparing the data to the theoretical implications of our assumptions.

We assume that the g-tensors of the interacting centers are identical (equivalent to assuming that both HiPIP molecules in the dimer are identical). Since direct (isotropic) exchange is not visible in EPR when the $g$-values of the interacting centers are the same [32], there must be a dimer orientation where the splitting from isotropic exchange is zero regardless of the relative orientations of the two g-tensors. For most other directions (crystal orientations) the isotropic splitting is nearly the same (isotropic). Since this orientation has an applied field direction relative to the g-tensor that is clearly not the direction denoted by the low-field resonances $\left(g_{7}\right)$ and since the splittings of the low-field region are not present in the axidl field region $\left(g_{\mathrm{x},}\right)$, then the low-field structure cannot result from an isotropic exchange interaction. Only the dipole-dipole interaction remains to account for the splittings.

The dipole-dipole interaction is twice as large when the intersystem vector is along the applied magnetic field than when the intersystem vector is perpendicular to the applied field. Referring to Fig. $7 \mathrm{~A}$, if the splitting in the low-field region denotes a direction that is perpendicular to the intersystem vector. then there must be a splitting in the high-field region that is twice as large as the splitting in the low-field region. Not only is this not the case, but any parameter set that has the low-field direction (in either spin system) more than ten degrees from the intersystem vector is incompatible with the data. We conclude that the intersystem vector in both spin systems is parallel to the direction of the g-tensor coordinate system corresponding to the low-field resonance (called the $z$-axis by us).

The arguments in the two preceding paragraphs require that the intersystem vector points in the same (within 10 degrees) direction relative to each of the cubanes. All of the interaction parameters for direction are therefore fixed except for the rotation of one protein relative to the other about the intersystem vector. Because the $g_{\mathrm{x}}$ and $g_{\mathrm{y}}$ values are nearly equal, we cannot specify this angle with any degree of certainty. However, we attempted to address this problem during the molecular modeling effort described below.

Another complexity presented by the spectra in Fig. 7 concerns the number of possible component spectra necessary to synthesize these experimental data. In studying Fig. 1. one can assume that all of the HiPIP molecules are paramagnetic at the beginning of the titration and that the spectra are linear combinations from monomers and dimers in "high salt'. For Fig. 2, there should be several combinations of HiPIP reduction represented by both dimers and monomers, but at the end of the titration all the molecules should be oxidized dimers. Because the spectrum at the end of the salt titration (Fig. 1E) is identical to that at the end of the redox titration (Fig. $2 \mathrm{H}$ ), we have assumed that Fig. 7A (that same spectrum) is characteristic of oxidized dimers only. The shape and resemblance of Fig. 1C. Fig. 2F and Fig. 3B in the low-field region argue that one of the dimers (component spectra) has this splitting in the low-field peak. This splitting corre- 
sponds to an intersystem distance of $16 \AA$ (see stick spectrat in Fig. 7). The rest of the features in the low-ficld region of Fig. $7 \mathrm{~A}$ can be added with another dimer (ai $1 / 2$ relative intencity) at $1.3 \AA$ intersystem distance and a slightly different $g$, value (see Table $D$ ) The huights of the sticks in Fig. 7 indicate the relative intensities of these resonances. In each of the stick patterns the high-ficld line of the pair is the more intense in contrast to the experimental data. A sum of two interaction spectra is the minimum number of components sufficient to generate the 'ramp.' The 'high salt monomer (Fig. 2A) is not a significant contributor to the ramp hecause its low-field maximum is halfway beiwcen the two low-field maxima in Fig. 7A. In other words, the ' $16 \AA$ dimer' and the 'high salt monomer' have the same $g_{-}$-values. On the other hand, the $13 \AA$ dimer' has a slightly modified $g$, Apparently the compression of the dimer is accompanied by a change in $g$. This change is explained in the following arguments

The fits shown in Fig. 7 are from identical parameters at both $X$ - and $Q$-band. The success of these fits ('vithout the minority component) at both trequencies argues for their uniqueness. Howeter, the conclusions are testable against the structural constraints from the published $X$-ray coordinates [8.9]. Inspection of the $P 2,2,2$, orthorhombic crystal packing reveals that the closest center-to-center distance of any two [4Fe-4S] clusters is over $22 \AA$. Neither dipole-dipole nor direct exchange interactions are expected to be significant at
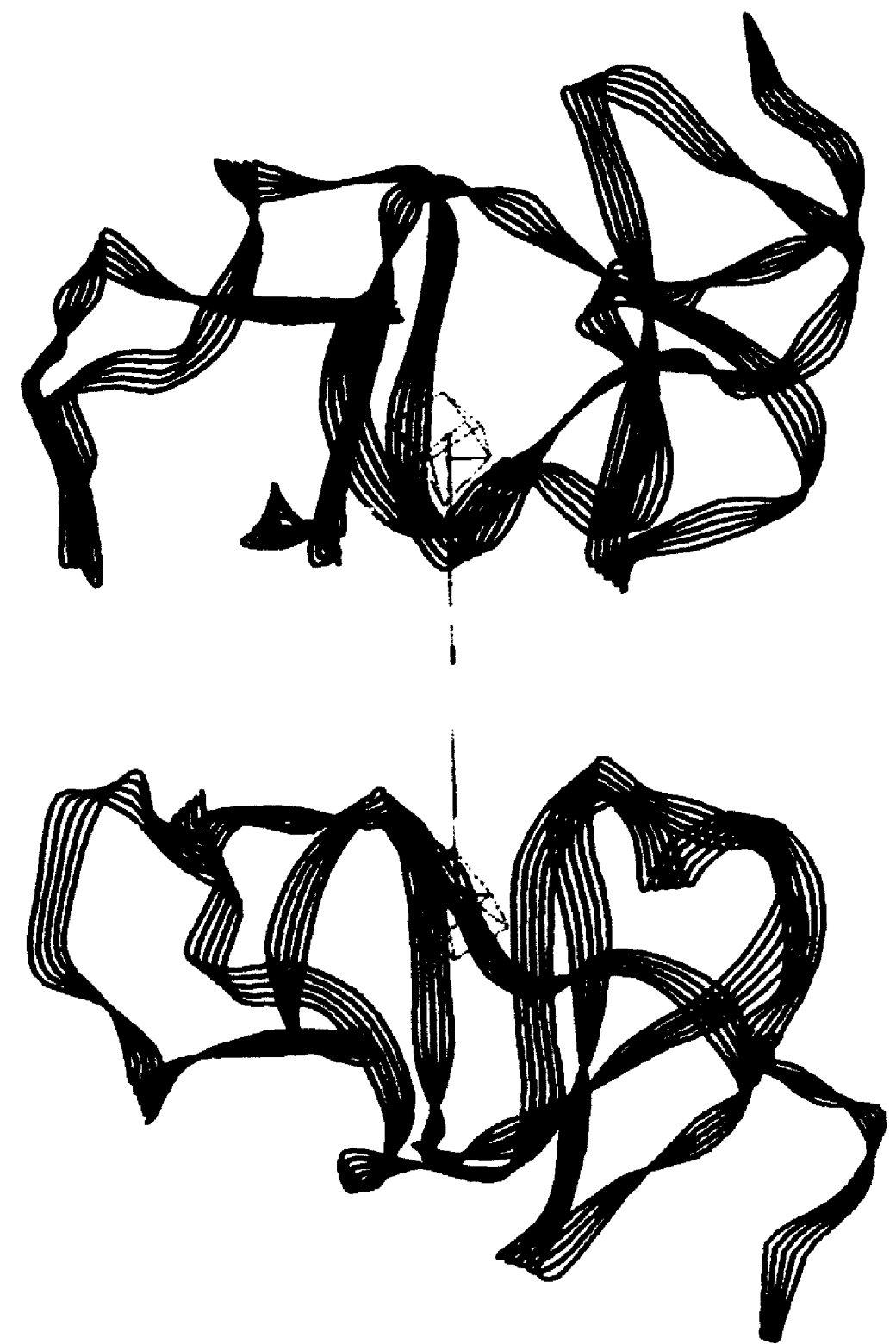

Fig. 8 . Model of the $C$. inesum HiPlP" high salt dimer synthesized from the work of Carter et al. [12.13]. The $\alpha$-carbon ribhon tracing of the 2 monomer units is represented in its final dimeric orientation along with the lwo cunanes apex to apex oricntation. $A$ if $\AA$ distance separates the centers of the Z cubanc and the entation angle of one unit relative w the other is approximately 44 degrees. 
these distances. There is however. it protein surface near one of the sulfur atoms in the $[4 \mathrm{Fe}-4 \mathrm{~S}]$ cluster. We therefore undertook molecular modeling studies to explore a hypothetical dimeric association that would be consistent with distances of 13 and $16 \AA$.

The dimeric $\alpha$-carbon ribbon tracing shown in Fig. 8 results from of our attempts to place two $C$. tinosum HiPIP molecules in Van de Waals contact with the cubane clusters as near as possible to one another and with the interciuster vector at the same direction relative to each of the cubanes. The molecules were rotated by 44 degrees about this vector to achieve a visual fit, but no attempt was made at energy minimization or freeing of the external residues from their crystal coordinates. The center-to-center distance of the two cubanes in Fig. 8 is $14 \AA$. Standard docking procedures (i.e., Van der Waals and electrostatic complementarity) were used to evaluate the selected surface interface. Because the dimer formation requires the presence of $\mathrm{NaCl}$ and freezing, we were hesitant to extend the $X$-ray crystallographic results to conclusions that included precise assumptions of side-chain charge. This interface is comparatively flat so that shifting the two molecules by one atom at their contact point increases the center-to-center distance $(3 \AA)$ as would the incorporation of a salt molecule or the rotation of a sidechain at the surface. The main point of Fig. $\delta$ is that the distances: 13 and $16 \AA$ are entirely consistent with this dimer structure.

Inspection of Fig. 8 also shows that one of the apices of the [4Fe- $\Delta \mathrm{S}]$ cubane is pointed at the nearby surface of the HiPIP molecule. The atom at this apex is a sulfur atom (S4, H86 (the cubane)). Since we have previously established that the direction from the cubane to the nearest surface is along the g-tensor $z$-axis, therefore the $z$-axis of the g-tensor is along the cubane 3-fold rotational axis through this sulfur atom. Furthermore, the $g$-value changes at $g$, (see Fig. 5) can be explained as resulting from distortions along this axis on the cubane caused by differences at the protein-water interface. Finally, the two forms of the 'high salt' $C$. rinosum HiPIP dimer can be envisioned as resulting from some difference at the protein-protein interface that changes the intercluster distance by $3 \AA$ and thercupon shifts $g_{z}$ very slightly (see Table I).

The emerging picture is one where $C$. l'inosum HiPIP molecules in high salt form two kinds of dimer upon freezing, which differ only slightly. A. minority torm of the protein also exists. but it is relat.vely insensitive to salt and solvent changes. Indeed, this EPR component may arise from a non-HiPIP protein; our data shed little light on the origin of this material other than its redox potential (see Fig. 2) and $g$-values (Table 1 ) are similar to those of HiPIP. Under the conditions where the dimer is not formed (all but $0.2-2 \mathrm{M} \mathrm{NaCl} C$. cinosum HiPIP freezes as a monomer (or a multimer in which the intercluster distance in greater than 20 A) whose EPR signal in the oxidized state is the same at $3, .$. but change, at $g$, depending on the whent $/ x$ lute composition. Thi unsitivity at $s$, is due $t o$ the fact that the z-axis of the cubane is normal to the protein surface and accessible to solvent and is therefore subject to changes brought about by the freazing process.

\section{Acknowledgements}

The Mossbauer spectra were collected with assistance from Mr. W.F. Filter. Preliminary work with $\mathrm{X}$-ray structure was provided by Ms. $\therefore$ nita Metzger with the advice of Professor Martha L'adwig. UM, Biophysics. This research was supported fi mancially hy the U.S. Public Health Service. GM 30974 and GM 35342 (JAF) and GM 12176 and 32785 (RHS).

\section{References}

I Lowenherg W. Ted. M1973) Iron-5ulfur Protems, Vols. 1 and 2. Academic Press. Vicu York.

2 Lovenhere. W. (ed.x1977) Iron-Sulfur Prolems, Vol 3. Aca. dernic Press. Nen York.

3 Spiro. T.G. ted.M1482 Iron-Sulfur Proteins. John Wiley $\&$ Sons. Nen lork.

4 Meyer. T.E.. Przysiecki. C.T.. Watkins. J.A. Bhattacharya. A. Simondwen. R.P.. Cusanowich. M.A. and Tollin. G. (1983) Proc. Natl. Acd. Sci. USA \$0. 67.41-67.41

5 Fukumori. T, and Yamanaka. T. $(1974)$ Current Microhiol. 3. $117-120$

6 Bartch. R.G. $(1963)$ in Bacterial Phowsynthesis (Gest. H. San Pielro. A and Vernon. L.P. eds). pp. 315-326. Antioch Press, Yellim Springs.

7 Dus. K. Tedro. S.M. and Bartuch. R.G. 1|973) 1. Biol Chem $34 \times .731 \times-7331$

s Carter. C.W.. Kraut. J., Freer. S.T. Xuang. Y.H. Alden. R.A and Bartact. R.G. 119741 J. Birl. Chem. 249. 4212-4225

9 Carter. C.W. Kraut, J. Freer. S.T. and Aluen. R.A. 194741 J Biol (hem. 249. 6.339-6.346

lil Tedro. S.M. Meyct. T.F. and Kamen. M.D. (1985) Arch Bickhem. Biophys. 211. nsts-thot.

I) Moms. T.H. Petering. D. and Palmer. G. (I969) J. Biol Chem 241. 2275-2277

12 Phillips. W.D. Poc. M. McDonalds. C. C and Bartwh. R.G

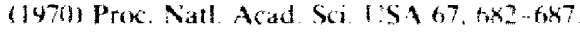

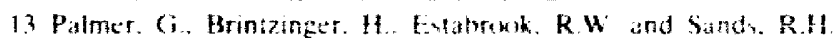
(1967) in Magnetic Rexonance in Biological Systems (Ehrenberg. A.. Malmstrom. B.G. and Vanngard. T.. eds.). pp. 159-17l. Pergamon Prese. London.

if Antanaitis. B.C. and Moss. T.H. (1475) Biochim. Biophys. Acta $405,262-279$

15 Evans. M.C.W. Hall. D.O. and Johnson. C.E. (1970) Biochem. I. 119. $289-291$

I6 Dickson. D.P.E. Johns C.E. Cammack. R. Evans. M.C.W Hall. D.O. and Rat. K.K. (1474) Binhem. J. 139. 1115-10K.

17 E.g.. Pfennig. $X$ and Widdel. F (1982) Phil. Trans. R. Soc Lond. B. 29x. $433-441$ or Plennig. N. (1467) Annu. Res. Microhiol 21. $285-324$.

IN Bartuch. RG (1971) Method Enamol 23, 644-649.

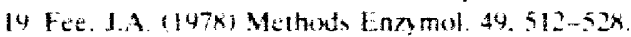


20) Bray.R.C. (1461) Biochem. 1.. 81. 189-193: Bray. R.C. and Pe1tersson. R. ihid, 194-195

21 Ballou. D P. and Palmer. G. (1974) Anal Chem. the 1246-1253,

22 Dunham. W.R., Wu. C.T., Polichar. R.M., Sands. R.H. and Haraing. L.J. (1977) Nucl. Instr. Meth. 145. 537\%553.

23 Filter, W.F. (198.3) Ph.D. Thesis. The University of Michigan

24 Dunham. W.R., Hagen. W.R., Braaksma. A., Grande, H.J. and Haaker. H. (1985) Eur. J Biochem. 14h. 497.501.

25 Newton. W.E. Ghellers. S.F.. Sands. R.H. and Dunham, W.R (1989) Biochem. Biophys. Res. Commun 162. $8 \times 2-841$.

26 Hagen. W.R.. Hearshen. D.O.. Sand, R.H. and Dunham. W.R (1985) J. Magn. Rewon. hl. 226)-2.32

27 Hagen. W.R. (1989) in Advanced EPR: Applications wo Biokgy and Biochemistry (Hoff. A.J.. ed.). (h. 22. Ehevier. Amsierdiam
Is Schepler. K.L. Dunham. W.R., Sands. R.H.. Fee, J.A. and Abeles, R.H. (1975) Biochim. Biophys. Acta 397. 510-518.

29 Sybyl 5.4 Iris 4D Version. Tripos Associates, Inc., 1699 South Hanley Rodd. Suite 303. St. Louis, MO. 63144.

30) Sands. R.H., Goldsmin. M.S.. Fee, J.A.. Antanaitis. B.C. and Moss. T.H. (1976) Abstract in the Proceedings of the VII International Conterence on Maguetic Resonance in Biological Systems. Montreal. Canada.

31 Middleton. P.. Dickson. D.P.E. Johnson. C.E. and Fush, J.D. (1980) Eur. J. Biochem.. 114, 289-29\%.

12 Pople. J.A.. Schneider. W.G. and Bernstein, H.J. (1999) High-resdution Nuclear Magnetic Resonance. McGraw-Hill. New York. p. 115 